\title{
Analysis of the Workflow Management System of Student Work
}

\author{
Yanping Chen \\ Information Engineering Department \\ Tianjin Bohai Vocational Technology College \\ TianJin, P.R.China \\ chenyanpingei@163.com
}

\author{
Bo Lin \\ Information Engineering Department \\ Tianjin Bohai Vocational Technology College \\ TianJin, P.R.China \\ linboei@163.com
}

\begin{abstract}
Through design and analysis, the student management system, the use of workflow technology. Workflow is a specific job into several tasks and task execution, string together through a series of specific rules and procedures, in order to achieve the work flow of information, standardization and automation. This paper describes in detail the application of workflow template, and eight kinds of workflow design in the system implementers. These executives basically meet the specific needs of the system, so as to improve the adaptability and flexibility of workflow.
\end{abstract}

Keywords- Students work in the workflow executor role

\section{INTRODUCTION OF THE MANAGEMENT SYSTEM OF STUDENT WORK}

With the deepening of the reform of higher education, school running scale constantly expanding, increasing the number of students in school, student management task has become increasingly heavy, the increasingly high demand. By using the method of the traditional manual management can not accurately understand the actual situation of students, not quick query student information, not for statistics and decision for the whole students, can not complete the student management affairs in time, affect the teacher to student's management ability and work level. Therefore, the design and the development of a student management system to the school's actual situation with.

Management system of student work mainly includes the student Party member database, student cadre database, database, student associations difficult student database, student discipline, student grants database database, student recruitment database, and provide the database for the information input, modify, delete, query function; provides statements printing function for the student information. The system also for different users with different permissions the user role, provides many kinds of workflow template convenient for teachers to complete a variety of student work, for example, development work, Party member of student activist Student Scholarship Award, outstanding student award the issuance of work, the new report job, graduates graduation work etc.

\section{APPLICATION OF WORKFLOW MANAGEMENT SYSTEM OF STUDENT WORK}

Workflow concept originated in the production and office automation systems, is the daily work with fixed procedures of the activities of a concept. Simply put, the workflow is a specific job into several tasks and task execution, string together through a series of specific rules and procedures, in order to achieve the enterprise process informatization, standardization and automation.

Through careful research and analysis, a lot of work flow management system of the student work in the need to realize the use of workflow technology. In the realization of the system design, first define the workflow template, which contains a transaction workflow execution of work, working conditions, and the need to deal with the object. Then during the operation of the system, according to the specific need to create an instance of the workflow template. For example, the new report workflow template, will be in the new born school report for each new to create a report of workflow instance. The workflow template independent of workflow instance running period, so based on the workflow instances of the same template can run at the same time. Each workflow workflow can be decomposed into multiple tasks, tasks can be divided into automatic task and artificial task two. Automatic completion of tasks is when meet the task when the trigger condition, the workflow server can automatically call some applications to complete the task. Artificial task refers to when meet the task when the trigger condition, the workflow server automatically send to objects and mail to the specified task executor inbox, a task executor artificial completed the task. In the workflow template for each artificial tasks designated executor, the performer belonging to the users of the system.

\section{STUDENT MANAGEMENT IN WORKFLOW EXECUTION MODEL}

In the process of research and the use of the workflow, workflow execution walker type setting is very important. The workflow executor mainly refers to the person or organization involved in the operation of the unit during task execution, implementation defined in the system affects the flexibility of workflow model. The system should provide users with a more perfect execution model, for the user to choose the workflow template design.

Through the analysis and the design of the project, summed up the system implementation in the workflow can be divided into the following eight categories:

\section{A. The workflow creator}

The workflow creator, or the administrator. Workflow server selects the workflow instances in the workflow 
instance begins when the administrator. In the absence of specified workflow instance is the default administrator, the workflow instance startup. The administrator can suspend, resume, resume, abort the workflow instance.

\section{B. The database owner}

The database owner. Workflow server specific user as the currently active database owner, all documents and data in the workflow instance execution time is saved by the database owner management.

\section{The specific user}

The specific user. In the creation of the workflow template to specify a specific user as a task executor.

Before the current task execution.

Before the current task execution. The performer's establishment, can make the workflow server from meet the current task trigger conditions users (that is, the ability to complete the task the user), choose to complete the task of recent users (i.e. the last completed the task of the workflow template user) as the current task execution.

\section{User group}

User group. Create user groups have similar roles users in the group. Through the establishment of user group, can realize the following three cases. The first case: create a workflow template select a group, after entering the workflow instance running period, the workflow server to each member of the group is assigned a separate task. That is to say, each member in the group should complete the task; second: create a template when selecting a group, during the workflow runtime, a member of the group to accept the task, the workflow server will delete the task automatically from other members of the group in the inbox; third situation: create a template when selecting a group, during the workflow runtime, workflow server determine which members of the group work load least allocates the members of this task.

\section{E. Authorized user}

Authorized user. When the original specified user is unable to complete the task, by original designation users specify additional users to complete the task.

\section{F. Be extended user}

Be extended user. When the original designated users found themselves unable to finish the task, can be extended, and the extended another user specified together to complete the task.

\section{G. In order to improve the adaptability and robustness of the workflow execution}

Role. In order to improve the adaptability and robustness of the workflow execution, specified in the workflow template execution, may specify a role, rather than a specific user or group. That is to say, the workflow template definition can be used when the role based access control. Role based access control. The basic idea is to assign a role to each task in the process based on, designate one or more users to each role, then the role authorization instead of user authorization. The workflow is used in this way can the task and role related, by assigning appropriate roles to users, the user and the task through role indirectly linked. Role is based on the system in order to complete various tasks and settings, user specific role is based on the powers and responsibilities of the user to distribution. So, the role of the middle layer can not put the user and task bound to die, effectively overcomes the shortcomings of the user and task will directly link. When add, delete or modify the user, for example the reality of the transferred, $\mathrm{V}$, job change, do not need to do a task executor change work, only the user role assignment, cancel, to achieve operational changes, greatly simplifies the task of executive management, reduce complexity, not error prone.

\section{SUMMARY}

In the design and implementation of student management system, extensive use of workflow technology, the performer as an important part of workflow, it is perfect, is flexible, is reasonable or not has great influence on the quality of the workflow. The main use of these eight kinds of implementation are introduced in detail in the paper. In the system, they can basically meet the specific needs of workflow, workflow to improve the adaptability and flexibility.

\section{REFERENCES}

[1] Coyne E J, Feinstein H L, Youman C E.Role based Access Control Models [J]. IEEE Computer, 1996, 29 (2):122-134.

[2] Workflow Management Coalition (WFMC). Workflow Process Definition Interface--XML Process Definition Language [S]. No. WFMC-TC-1025, Version 1.02002.

[3] Fan Yushun. Basis of workflow management technology -- the implementation of business process reengineering and business process automation core technology [M]. Beijing: Tsinghua University press, 2001

[4] Zhang Ying, "looks and implementation of Master Thesis], the design of student management information system of institutions of higher learning $(2010,03)$ 